- Nasopharyngeal Carcinoma Column.

\title{
Regulation of hypoxia-induced mRNA expressions of HIF-1 $\alpha$ and osteopontin and in vitro radiosensitization by tirapazamine in human nasopharyngeal carcinoma HNE-1 and CNE-1 cells
}

\author{
Peng $\mathrm{Xu}^{1}$, Jian-Ming Huang ${ }^{2}$, Yuan Ren ${ }^{2}$, Xiao Zha², Bi-Fang Deng' ${ }^{2}$ Jun-Hui Wu², Jin-Yi Lang ${ }^{2}$ \\ ${ }^{1}$ Guangxi Medical University, Nanning, Guangxi 530021, P. R. China; ${ }^{2}$ Sichuan Cancer Hospital, Chengdu, Sichuan 610041, P. R. China
}

\begin{abstract}
[Abstract] Background and Objective: Combined hypoxic cytotoxic drugs and chemoradiotherapy is an important mean of oncotherapy, and Tirapazamine (TPZ) is one of the most remarkable drugs. It has been shown that TPZ has a synergistic effect with radiotherapy on tumor cells, but whether TPZ would down-regulate the expression of the hypoxia-induced genes has not been reported. This study was to investigate the hypoxia-induced mRNA expressions of hypoxia inducible factor-1 $\alpha$ (HIF-1 $\alpha)$ and osteopontin (OPN) in human nasopharyngeal carcinoma HNE-1 and CNE-1 cells and the radiosensitization of TPZ, a hypoxiaspecific drug, on HNE-1 and CNE-1 cells in vitro. Methods: The IC ${ }_{50}$ values of TPZ for HNE-1 and CNE-1 cells were measured using MTT assay, and the mRNA expressions of HIF-1 $\alpha$ and OPN in HNE-1 and CNE-1 cells was determined using RT-PCR under aerobic and hypoxic conditions, respectively. The survival rates of HNE-1 and CNE-1 cells treated with or without TPZ at $\mathrm{IC}_{10}$ in the presence or absence of oxygen for $6 \mathrm{~h}$ were determined using colony formation assay following exposure to 1-6 Gy of ${ }^{60} \mathrm{Co}$ radiation. The dose-survival curves were plotted and the values of DO, Dq and SER were calculated as a single-hit multitarget model. Results: The $I_{50}$ values of TPZ were $34.81 \mu \mathrm{mol} / \mathrm{L}$ and $35.02 \mu \mathrm{mol} / \mathrm{L}$ in $\mathrm{HNE}-1$ and $\mathrm{CNE}-1$ cells under aerobic condition, and $30.20 \mu \mathrm{mol} / \mathrm{L}$ and $28.48 \mu \mathrm{mol} / \mathrm{L}$ under hypoxic condition, respectively. The expressions of HIF-1 $\alpha$ and $\mathrm{OPN}$ mRNA were reduced by TPZ in HNE-1 cells, but not in CNE-1 cells under hypoxic condition. For the HNE-1 cells, the respective values of Do and Dq were $0.89 \mathrm{~Gy}$ and 0.28 Gy following normoxic irradiation versus 1.47 Gy and 0.44 Gy following hypoxic irradiation. For the CNE-1 cells, the respective values of DO and Dq were 0.72 Gy and 0.68 Gy following normoxic irradiation versus $0.95 \mathrm{~Gy}$ and $0.56 \mathrm{~Gy}$ following hypoxic irradiation. The values of DO and Dq for HNE-1 and CNE-1 cells treated with TPZ under hypoxic condition following irradiation were $0.66 \mathrm{~Gy}, 0.21 \mathrm{~Gy}$ and $0.85 \mathrm{~Gy}, 0.79 \mathrm{~Gy}$, respectively. Conclusion: TPZ can down-regulate hypoxia-induced expression of HIF-1 $\alpha$ and OPN mRNA of HNE-1 cells and radiosensitize the HNE-1 cells but not CNE-1 cells, and act as a hypoxia modifier.
\end{abstract}

Key words: Tirapazamine, nasopharyngeal carcinoma, irradiation, HIF-1 $\propto$, osteopontin

Tumor hypoxia is one of the main reasons for radiotherapy and chemotherapy failure in solid tumors (chemotherapy and radiotherapy resistance). The hypoxic cells account for $10 \%-50 \%$ in solid tumors and their tolerance to radiation and chemotherapy is 2.5-3 times stronger than that of aerobic cells, which becomes one of the important factors making cancer difficult to cure, and easy to recur and metastasize. Therefore, the toxic drugs to

Correspondence to: Jin-Yi Lang; Tel: +86-28-85420173; Email: langjy@tfol.com This paper was translated from Chinese into English by CJC Medical Translation and edited by Jing-Yun Ma on 2009-11-14.

The Chinese version of this paper is avaiable at http://www.cjcsysu.cn/cn/article .asp?id $=16518$

Received: 2009-07-07; Accepted: 2009-10-16

Grant: Research Foundation of the Science and Technology Department of Sichuan Province (No. 2008JY0020)

126 hypoxic cells in combination with chemoradiotherapy is an important regimen for cancer treatment. Tirapazamine (TPZ) is one of the most remarkable toxic drugs to hypoxic cells. TPZ enters into the cell, forms a high activity of free radicals through the role of intracellular reductase, and reduces the intracellular oxygen ion into oxygen. Under hypoxic condition, the highly active TPZ free radical can obtain a hydrogen atom from the DNA through the mediation of topoisomerase II, cause single/double-strand breaks and chromosome damage, thus resulting in cell death. Studies have shown that TPZ combined with radiotherapy has synergistic effects on tumor cells, but whether it can down-regulate hypoxia-induced genes under hypoxic condition has not been reported ${ }^{1-3}$. In this experiment, $\mathrm{HNE}-1^{\mathrm{EB}+}$ and $\mathrm{CNE}-1^{\mathrm{EB}-}$ human nasopharyngeal carcinoma cells were used as an in vitro model to explore the expression differences of hypoxia inducible factor-la (HIF-1a) and OPN in these two cell lines under normoxic and hypoxic conditions and 2010; Vol.29 Issue 2 


\section{Chinese Journal of Cancer}

the impact of TPZ on the expression, and to detect the radiation sensitizing effects of TPZ on the two nasopharyngeal carcinoma cell lines under hypoxic condition by colony-forming method so as to provide a new sensitizing means for clinical radiotherapy of nasopharyngeal carcinoma.

\section{Materials and methods}

\section{Drugs and reagents}

TPZ was purchased from the U.S. Biotechnology Co., Ltd.; methlthiazolyl tetrazolium (MTT) and dimethyl sulfoxide (DMSO) from Sigma; RPMI-1640 medium from Gibco; calf serum from China Lanzhou Ming-Hai Bio-Engineering Company; Trizol kit from Shanghai Bio-Engineering Co., Ltd.; and RT-PCR kit from Chengdu Boracker Biotechnology Co., Ltd.

\section{Cells and culture conditions}

Human nasopharyngeal carcinoma cell lines HNE-1 (EBV-positive) and CNE-1 (EBV-negative) were obtained from the Sichuan Provincial Tumor Institute and were cultured in RPMI-1640 culture medium containing fetal calf serum (10\%), penicillin $(100 \mathrm{u} / \mathrm{mL})$ and gentamicin $(40 \mathrm{u} / \mathrm{mL})$ under conventional conditions $\left(37^{\circ} \mathrm{C}, 5 \% \mathrm{CO}_{2}\right)$; for hypoxic culture, conventionally cultured cells were placed in a hypoxic culture device containing $5 \% \mathrm{CO}_{2}$ and $0.01 \% \mathrm{O}_{2}$. All experiments were carried out in the cell exponential growth phase.

\section{Detection of cell growth inhibition by MTT}

The HNE-1 and CNE-1 cells in exponential growth phase were collected, adjusted to a cell concentration of $5 \times 10^{4} / \mathrm{mL}$ with RPMI-1640 medium containing $10 \%$ fetal calf serum, and were inoculated onto a 96-well plate, $0.1 \mathrm{~mL} /$ well. After cell adherence, different concentrations of TPZ (a final concentration of 5-50 $\mu \mathrm{mol} / \mathrm{L}$ ) were added, the drug of each concentration was inoculated into four holes. After $72 \mathrm{~h}$ under normoxic and hypoxic conditions, $20 \mu \mathrm{L}$ MTT $(5 \mathrm{mg} / \mathrm{mL})$ was added, supernatant was discarded after $4 \mathrm{~h}$ incubation at $37^{\circ} \mathrm{C}$, and added with $150 \mu \mathrm{L}$ dimethyl sulfoxide (DMSO). After full blending, DF-M3000 enzyme-labeled instrument was used to measure the absorbance ( $A$ value) at $570 \mathrm{~nm}$ wavelength and the cell survival rate was calculated by the following formula, as well as the concentration-survival regression equation and half-effective inhibitory concentration $\mathrm{IC}_{50}$ and $\mathrm{IC}_{10}$. Each experiment was repeated 3 times.

Cell survival rate $=\left(A_{\text {test group }}-A_{\text {blank group }}\right) /\left(A_{\text {control group }}-A_{\text {blank group }}\right) \times 100 \%$ Detection of HIF-1 $\alpha$ and OPN expression by RT-PCR

The HNE-1 and CNE-1 cells treated or untreated by TPZ and cultured under hypoxic or normoxic conditions were collected, the medium was discarded, washed by PBS for twice and added with $1 \mathrm{~mL}$ Trizol reagent. The total RNA was extracted according to the instructions from Trizol kit, and quantitatively examined by $1.5 \%$ agarose gel electrophoresis, which showed two brightness diminishing clear bands of $28 \mathrm{~S}$ and $18 \mathrm{~S}$ without obvious sign of dispersion, and the brightness ratio of $28 \mathrm{~S}$ and $18 \mathrm{~S}$ band was about $2: 1$. The total RNA $(2 \mu \mathrm{g})$ with a final reaction volume of 20 $\mu \mathrm{L}$ was reversibly transcribed into cDNA. Two $\mu \mathrm{L}$ cDNA was used for PCR with $\beta$-actin as internal reference, and the PCR primers were designed using Primer premier 4.0 software. $\beta$-actin primers: 5'-AAGAGATGGCCACGGCTGCT-3' for upstream primer, and 5'-GACTCGTCATACTCCTGCTTGCT-3' for downstream primer (421 bp); HIF-1a primers: 5'-TTGATTGCATCTCCATCTCCT-3' for upstream primer, and 5'-TTCGCTTTCTCTGAGCATTCT-3' for downstream primer (249 bp); OPN primers: 5'-GGAGGAG GCAGAGCACAG-3' for upstream primer, 5'-CGTTGGACT TACTTGGAAGG-3' for downstream primer (331 bp). HIF-1a reaction conditions: $94^{\circ} \mathrm{C}$ for $2 \mathrm{~min}$, followed by 35 cycles of $94^{\circ} \mathrm{C}$ for $0.5 \mathrm{~min}, 56^{\circ} \mathrm{C}$ for $0.5 \mathrm{~min}$ and $72^{\circ} \mathrm{C}$ for $1 \mathrm{~min}$, and then thermal insulation at $22^{\circ} \mathrm{C}$. OPN reaction conditions: $94^{\circ} \mathrm{C}$ for 2 $\mathrm{min}$, followed by 35 cycles of $94^{\circ} \mathrm{C}$ for $0.5 \mathrm{~min}, 55^{\circ} \mathrm{C}$ for $0.5 \mathrm{~min}$ and $72^{\circ} \mathrm{C}$ for $1 \mathrm{~min}$, and then thermal insulation at $22^{\circ} \mathrm{C}$. $\beta$-actin reaction conditions were the same with HIF- $1 \alpha$ and OPN. Six $\mu \mathrm{L}$ PCR product was used for $1.5 \%$ agarose gel electrophoresis, the gray stripe was measured quantitatively by ultraviolet imaging system, and the ratio of HIF- $1 \alpha$ and OPN to $\beta$-actin was analyzed respectively. Each experiment was repeated four times.

\section{Irradiation method and dose-survival curve}

${ }^{60} \mathrm{Co} \gamma$-ray was used with an open-field size of $5 \mathrm{~cm} \times 5 \mathrm{~cm}$, a depth of $80 \mathrm{~cm}$ (SSD) and a liquid depth of $0.5 \mathrm{~cm}$. Radiation doses were $0,0.2,0.4,1,2,3,4,5$ and $6 \mathrm{~Gy}$. The HNE-1 and CNE-1 cells in exponential growth phase were collected and inoculated into 12-well plates, $2.5 \times 10^{5}$ cells/well, added with TPZ $\left(\mathrm{IC}_{10}\right)$, and irradiated after $6 \mathrm{~h}$ under normoxic and hypoxic conditions. After irradiation, the cells were collected immediately with $0.25 \%$ trypsin-EDTA, counted and inoculated at a $60 \mathrm{~mm}$ dish in diameter by adding $4 \mathrm{~mL}$ RPMI-1640 medium containing $10 \%$ fetal calf serum, cultured for 14 days at $37^{\circ} \mathrm{C}, 5 \% \mathrm{CO}_{2}$, the medium was discarded and stained by solution with crystal violet $(0.5 \%)$ and methanol $(30 \%)$ for $1 \mathrm{~min}$, and then the clone numbers in each dose group were counted (for colonies with more than 50 cells) and the cell survival fraction (SF) was calculated. The experiment was repeated three times. The single-hit multi-target mathematical model was used to draw a dose-survival curve, D0, Dq, SF2 and radiosensitization ratios (SER) were obtained, and the radiosensitivity of each group was analyzed and compared.

\section{Statistical analysis}

The experimental data were expressed as mean \pm SD and tested by $t$ test. Analysis of variance was used to compare two groups. SPSS13.0 statistical software was used and the test level was 0.05 .

\section{Results}

\section{Growth inhibition of nasopharyngeal carcinoma cell lines by TPZ}

The $\mathrm{IC}_{50}$ of TPZ in HNE-1 and CNE-1 cells under normoxic condition was $(34.81 \pm 4.18) \mu \mathrm{mol} / \mathrm{L}$ and $(35.02 \pm 4.20) \mu \mathrm{mol} / \mathrm{L}$; the $\mathrm{IC}_{50}$ in HNE-1 and CNE-1 cells under hypoxia condition was $(30.20 \pm 3.32) \mu \mathrm{mol} / \mathrm{L}$ and $(28.48 \pm 3.13) \mu \mathrm{mol} / \mathrm{L}$, respectively. Compared with normoxic group serving as control, the sensitivity to TPZ was increased in hypoxic groups and the $\mathrm{IC}_{50}$ ratio was 
0.87 and 0.81 , respectively.

\section{Expressions of HIF-1 $\alpha$ and OPN in HNE-1 and CNE-1 nasopharyngeal carcinoma cell lines}

Semi-quantitative RT-PCR test showed that under normoxic condition, HIF-1a mRNA was expressed in two kinds of nasopharyngeal carcinoma cells, but at low level and the expression was cell-specific, which was higher in CNE-1 cells. After culture under hypoxic condition for $24 \mathrm{~h}$, the expression of HIF-1a mRNA significantly increased in HNE-1 cells, but slightly decreased in CNE-1 cells. After treatment by different concentrations of TPZ, the expression level in HNE-1 cells became lower than in the hypoxic group, but without significant dependency on concentration, while that in CNE-1 cells increased, as shown in Figures 1 and 2.

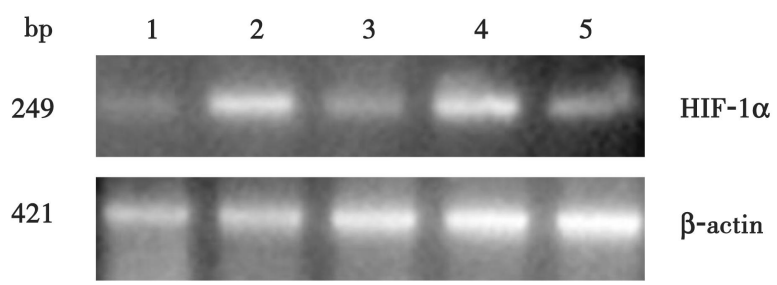

Figure 1 The expression of HIF-1 $\propto$ mRNAs of HNE-1 cells under different conditions

1, aerobic condition; 2, hypoxic condition; 3, hypoxic condition and $0.2 \mu \mathrm{mol} / \mathrm{L}$ TPZ treatment; 4, hypoxic condition and $2 \mu \mathrm{mol} / \mathrm{L}$ TPZ treatment; 5, hypoxic condition and $20 \mu \mathrm{mol} / \mathrm{L}$ TPZ treatment.

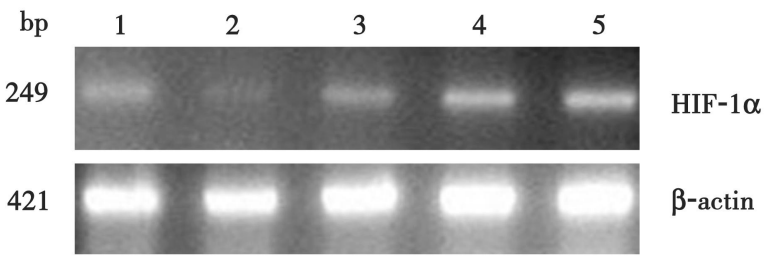

Figure 2 The expression of HIF-1 $\propto$ mRNAs of CNE-1 cells under different conditions

1, aerobic condition; 2, hypoxic condition; 3 , hypoxic condition and $0.2 \mu \mathrm{mol} / \mathrm{L}$ TPZ treatment; 4, hypoxic condition and $2 \mu \mathrm{mol} / \mathrm{L}$ TPZ treatment; 5, hypoxic condition and $20 \mu \mathrm{mol} / \mathrm{L}$ TPZ treatment.

Under normoxic condition, OPN mRNA was expressed in HNE-1 cells at a very low level and was not expressed in CNE-1 cells. Under hypoxic condition, it was expressed in two kinds of nasopharyngeal carcinoma cells at a significantly increased level, especially in HNE-1 cells. After treatment with different concentrations of TPZ, the expression levels in the two kinds of cells gradually decreased with increased concentration as compared with those in hypoxia group (Figures 3 and 4).

\section{TPZ radiosensitization}

The HNE-1 cell line had a D0 value of $0.89 \mathrm{~Gy}$ and a Dq value of $0.28 \mathrm{~Gy}$ in normoxic irradiation group, a D0 value of 1.47 Gy and a Dq value of $0.44 \mathrm{~Gy}$ in hypoxic irradiation group, and a $\mathrm{D} 0$ value of $0.66 \mathrm{~Gy}$ and a $\mathrm{Dq}$ value of $0.21 \mathrm{~Gy}$ in hypoxic

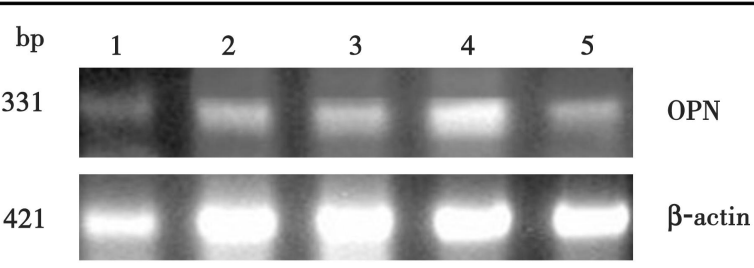

Figure 3 The exp ression of OPN mRNAs of HNE-1 cells under different conditions

1 , aerobic condition; 2, hypoxic condition; 3 , hypoxic condition and $0.2 \mu \mathrm{mol} / \mathrm{L}$ TPZ treatment; 4, hypoxic condition and $2 \mu \mathrm{mol} / \mathrm{L}$ TPZ treatment; 5 , hypoxic condition and $20 \mu \mathrm{mol} / \mathrm{L} \mathrm{TPZ}$ treatment.

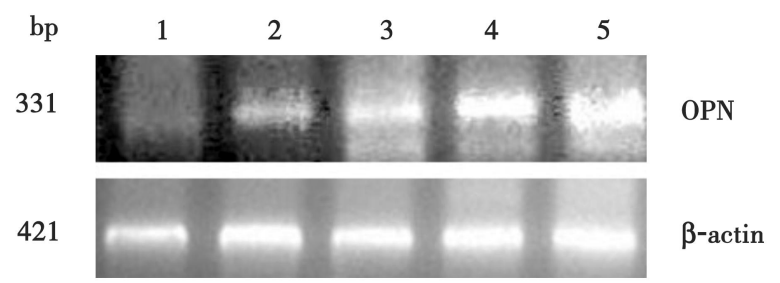

Figure 4 The exp ression of OPN mRNAs of CNE-1 cells under different conditions

1, aerobic condition; 2, hypoxic condition; 3 , hypoxic condition and $0.2 \mu \mathrm{mol} /$ L TPZ treatment; 4, hypoxic condition and $2 \mu \mathrm{mol} / \mathrm{L}$ TPZ treatment; 5 , hypoxic condition and $20 \mu \mathrm{mol} / \mathrm{L}$ TPZ treatment.

drug-added irradiation group. The CNE-1 cell line had a D0 value of $0.72 \mathrm{~Gy}$ and a $\mathrm{Dq}$ value of $0.68 \mathrm{~Gy}$ in normoxic irradiation group, a D0 value of $0.95 \mathrm{~Gy}$ and a Dq value of $0.56 \mathrm{~Gy}$ in hypoxic irradiation group, and $a D_{0}$ value of $0.85 \mathrm{~Gy}$ and a $\mathrm{Dq}$ value of $0.79 \mathrm{~Gy}$ in hypoxic drug-added irradiation group. Figures 5 and 6 showed the radiation survival curves of HNE-1 and CNE-1 in normoxic group, hypoxic group and hypoxia drug-added group, respectively. It could be seen that HNE-1 cells had a significantly lower radiosensitivity after treatment by hypoxia ( $P$ $<0.05$ ), while the radiosensitivity significantly increased after TPZ treatment $(P<0.05)$; CNE-1 cells had a slightly lower radiosensitivity after hypoxic treatment $(P>0.05)$, while the radiosensitivity slightly increased after TPZ administration $(P>$ $0.05)$.

\section{Discussion}

In this study, the results showed that under hypoxic condition, TPZ could enhance the cytotoxicity in HNE-1 and CNE-1 cells, especially in HNE-1 cells. Studies have shown that TPZ enters into the cell, forms a highly active free radical through the action of intracellular reductase, and the intermediate of free radical is rapidly oxidized into the parent molecule under aerobic condition, while producing a super-oxide free base, leading to occurrence of side effects such as muscle spasm when patients receive TPZ treatment. Under hypoxic condition, the highly active TPZ free radical can obtain a hydrogen atom from adjacent large molecule, resulting in DNA single/double-strand breaks and chromosome damage mediated by topoisomerase II, and causing cell death. In the hypoxic microenvironment in solid tumors, tumor cells maintain their metabolic stability and promote their growth and

2010; Vol.29 Issue 2 


\section{Chinese Journal of Cancer}

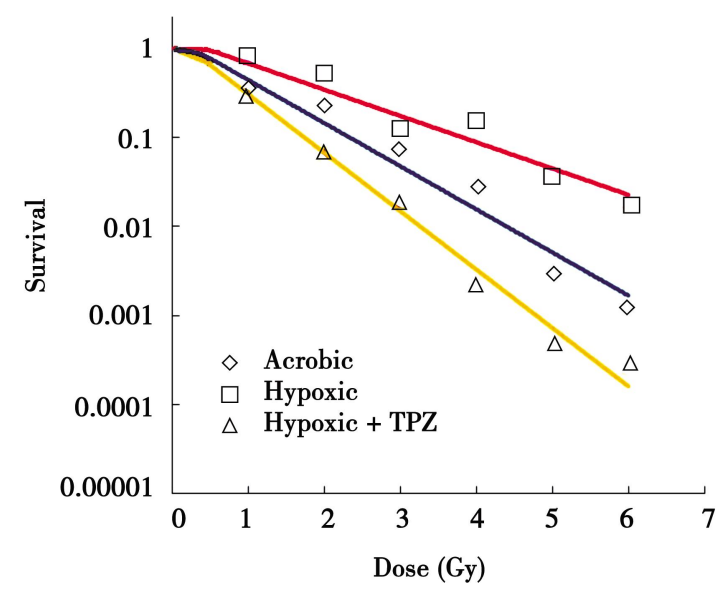

Figure 5 The dose-survival curves of different groups of HNE-1 cells fitted by a single-hit multitarget model

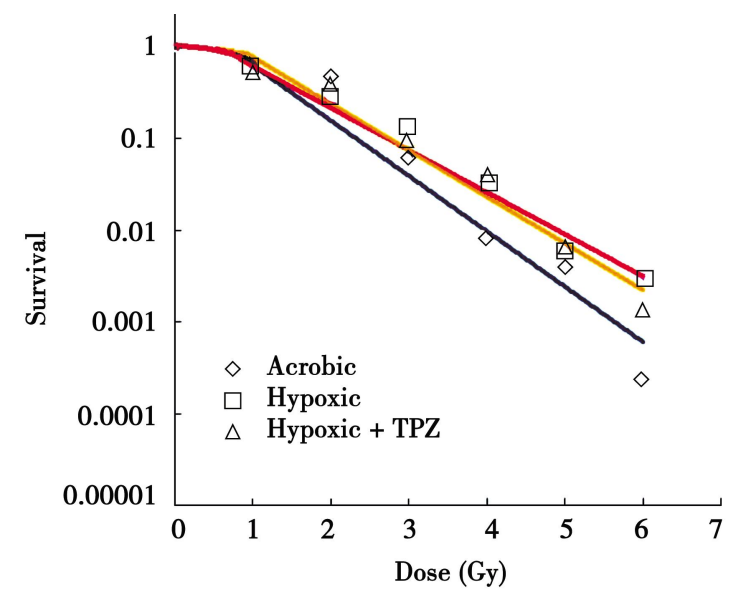

Figure 6 The dose-survival curves of different groups of CNE-1 cells fitted by a single-hit multitarget model

metastasis through the overexpression of a specific transcription factor-HIF-1 $\alpha$. Through regulating the expression of genes related to hypoxia, HIF-1 $\alpha$ plays an important role in the pathophysiology maintaining oxygen homeostasis in the body. Some of the target genes and proteins regulated by HIF-1 $\alpha$ are directly or indirectly related to apoptosis, among which there are factors that promote cell apoptosis, but also factors that inhibit apoptosis, so it is these factors that determine the HIF-1 $\alpha$ and play pro-apoptotic and anti-apoptotic dual regulatory roles. On one hand, HIF-1 $\alpha$ plays an anti-apoptotic role by increasing anaerobic metabolism and glucose extraction and reducing pro-apoptotic gene expression ${ }^{4,5}$; on the other hand, it promotes apoptosis of tumor cells through inhibiting the degradation of wild-type P53 protein and stabilize it to elevate its level. In this study, under hypoxic condition, the HIF-1 $\alpha$ expression in HNE-1 cell line significantly increased because the EB virus could promote HIF-1 $\alpha$ expression through latent membrane protein 1 $(\text { LMP1 })^{6,7}$. After the use of TPZ, it was decreased probably because under hypoxic condition, through the role of intracellular reductase, TPZ formed a highly active free radical while producing oxygen, and down-regulating the HIF-1 $\alpha$ expression by increasing the oxygen concentration of cell micro-environment.
OPN is also a hypoxia-related gene frequently studied in recent years. Hypoxia can induce OPN transcription, and this process is associated with the activation of AKT signaling pathway ${ }^{8}$. By enzyme-linked immunosorbent assay and micro-electrode method, Le et al. ${ }^{9}$ found in patients with advanced head and neck squamous cell carcinoma, the OPN level in plasma was negatively correlated with the oxygen partial pressure in tumor tissues. In this study, TPZ also down-regulated OPN expression under hypoxic condition, which further proved that TPZ could improve the oxygen concentration of cell micro-environment.

Tumor hypoxia has been one of the main factors compromising the efficacy of radiotherapy and chemotherapy. Under hypoxic condition, on one hand, the radiation-generated oxygen free radicals in tumor cells are reduced, lessening the radiotherapy-induced DNA breakage; on the other hand, HIF-1 is specifically over-expressed, increases the expression of vascular endothelial growth factor (VEGF), and promotes tumor proliferation, resulting in decreased radiosensitivity. Many previous studies using TPZ for hypoxic cells have shown that TPZ can enhance the radiosensitivity of hypoxic cells ${ }^{10-13}$. Our study on radiosensitivity of HNE-1 and CNE-1 cells under normoxic and hypoxic conditions confirmed that, under hypoxic condition, the radiosensitivity of both nasopharyngeal carcinoma cells decreased, especially the HNE-1 cells, which was similar to the results from most of the other clinical reports. The increased radiosensitivity after the use of TPZ under hypoxic condition may be due to the increased oxygen concentration of cell micro-environment and the inhibition of HIF-1a expression.

In this study, for the first time, TPZ is found to up-regulate the mRNA expressions of hypoxia-related gene HIF-1 $\alpha$ and OPN, while enhancing the radiosensitivity through increasing the oxygen concentration in cell micro-environment, so it can be used as a kind of oxygen additive to increase the radiosensitivity of human nasopharyngeal carcinoma hypoxic cell lines. However, the role of TPZ in the protein expression of hypoxia-related gene HIF-1 $\alpha$ and OPN, as well as downstream proteins needs to be further studied. At present, this drug is in phase III clinical trials abroad, but part of the clinical trials show that TPZ has obvious side effects such as muscle spasm, therefore, how to further overcome its toxicity and target hypoxic cells is still a focus of therapy targeting tumor hypoxia.

\section{References}

[1] Lartigau E, Guichard M. Does tirapazamine (SR-4233) have any cytotoxic or sensitizing effect on three human tumour cell lines at clinically relevant partial oxygen pressure? [J].Int J Radiat Biol, 1995,67(2): 211-216.

[2] Zhang M, Stevens G. Effect of radiation and tirapazamine (SR-4233) on three melanoma cell line [J]. Melanoma Res, 1998,8(6): 510-515.

[3] Lambin P, Guichard M, Chavaudra N, et al. The effect of the hypoxic cell drug SR-4233 alone or combined with the ionizing radiations on two human tumor cell lines having differen radiosensitivity [J]. Radiother Oncol, 1992,24(3): 201-204

[4] Zaman K, Ryu H, Hall D, et al. Protection from oxidative stress-induced apoptosis in cortical neuronal cultures by iron chelators is associated with enhanced DNA binding of hypoxia-inducible factor-1 and ATF-1/CREB and increased expression of glycolytic enzymes, p21 (waf1/cip1), and erythropoietin [J]. J Neurosci, 1999,19(22):9821-9830.

[5] Erler JT, Cawthorne CJ, Williams KJ, et al. Hypoxia-mediated down- 


\section{Chinese Journal of Cancer}

regulation of bid and bax in tumors occurs via hypoxia-inducible factor 1 dependent and -independent mechanisms and contributes to drug resistance [J]. Mol Cell Biol, 2004,24(7):2875-2889.

[6] Wakisaka N, Kondo S, Yoshizaki T, et al. Epstein-Barr virus latent membrane protein 1 induces synthesis of hypoxia-inducible factor $1 \alpha[\mathrm{J}]$. Mol Cell Biol, 2004,24(12):5223-5234

[7] Kondo S, Seo SY, Yoshizaki T, et al. EBV latent membrane protein 1 upregulates hypoxia-inducible factor 1alpha through Siah1-mediated downregulation of prolyl hydroxylases 1 and 3 in nasopharyngeal epithelial cells [J]. Cancer Res, 2006, 66(20):9870-9877.

[8] Zhu Y, Denhardt DT, Cao H. Hypoxia upregulates osteopontin expression in NIH-3T3 cells via a Ras-activated enhancer [J]. Oncogene, 2005, 24 (43):6555-6563.

[9] Le Q T, Sutphin PD, Rayehaudhufi S, et al. Identification of osteopontin as a prognostic plasma marker for head and neck squamous cell carcinomas [J]. Clin Cancer Res, 2003, 9(1):59-67.

[10] Masunaga S, Ono K, Hori H, et al. Effects of bioreductive agents tirapazamine and mitomycin $\mathrm{C}$, on quiescent cell populations in solid tumors, evaluated by micronucleus assay [J]. Jpn J Cancer Res, 1997,88 (9): 907-914

[11] Masunaga $\mathrm{S}$, Ono $\mathrm{K}$, Hori $\mathrm{H}$, et al. Change in oxygenation status in intratumour total and quiescent cells following gamma-ray irradiation, tirapazamine administration, cisplatin injection and bleomycin treatment [J]. Br J Radiol, 2000,73(873): 978-986.

[12] Masunaga S, Ono $\mathrm{K}, \mathrm{Hori} \mathrm{H}$, et al. Potentially lethal damage repair by total and quiescent tumor cells following various DNA damaging treatments [J]. Radiat Med, 1999,17(4): 259-264.

[13] Beck R, Röper B, Carlsen JM, et al. Pretreatment 18F-FAZA PET predicts success of hypoxia-directed radiochemotherapy using tirapazamine [J]. J Nucl Med, 2007,48(6):973-980. 